\title{
Timely Public Health Intervention and Vectorborne Response Planning
}

\section{Megan Holderness}

\author{
Epidemiology, OKC-Co Health Department, Oklahoma City, Oklahoma, United States
}

Objective

Demonstrate the impact of surveillance and media engagement on Public Health protection during a Vectorborne disease response. Identify surveillance and reporting methods for timely response to positive cases steps. Explore and apply best practices for collaboration with partners and surrounding municipalities in order to reduce disease impact

\section{Introduction}

The OKC-Co Health Department deployed a phased vectorborne response plan to address multiple diseases, including Zika Virus and West Nile Virus. This plan is scalable and flexible, but must necessarily prepare for the "worst case" scenario. Although not currently a local threat in OKC-Co, Zika virus response planning requires early coordination between state, local and federal agencies in order to mitigate risk to the population. The backbone of the Vectorborne response planning has been proven successful through West Nile Virus response in which Oklahoma has experienced three outbreak seasons: 2003, 2007 and 2012. (OSDH) In 2015, the OKC area experienced a greater than $112 \%$ increase in the number of vectors and 18 WNV positive test pools were observed. The heightened number of vectors and positive test pools did not translate to the same escalation in human cases, which demonstrates the strength that Public Health collaboration between surrounding municipalities and community members has on reducing the potential impact of this seasonal epidemic. During the most recent 2017 mosquito season, local code enforcement, city officials and consumer protection deployed a total of 18 CDC Gravid and BG Sentinel traps. The final day of sorting took place during the last week of October, as consistent with the decrease in mosquito numbers. There were 23 trapping and testing weeks with a total of 43,079 mosquitos trapped and 33, 846 mosquitos tested. An average of $66 \%$ of all trapped mosquitos were tested each week. The Maximum Likelihood Estimation (MLE) Infection Rate is calculated each week.

\section{Methods}

The OKC-Co Health Department implements a multi-level approach to communication, prevention, surveillance and disease abatement Vectorborne disease. This approach includes:

-Hazard assessment: Vector activity, disease risk and mitigation strategies are included in the response plan and discussed with partners as needed.

-Media outreach

-Vector surveillance: OCCHD conducts local vector surveillance by trapping, lab species identification and testing for West Nile Virus. The plans also provide testing capabilities in partnership with State and Federal agencies. Trapping is accomplished using CDC Gravid Traps and BG Sentinel Traps. Trapped mosquitos are brought to the laboratory at OCCHD on a weekly basis and are frozen overnight. The following morning, mosquito genus and species are identified and vector species are tested.

-Environmental treatments: Consumer Protection identifies and treats areas with stagnant water following a complaint.

-Habitat remediation: OCCHD and its partners have resources and coordination planned for habitat remediation events

-Municipality partnerships: OKCSWQ sends OCCHD weekly reports for each of the trap sites that includes information on trap condition, needed repairs, and weekly mosquito count. OCCHD provides larvicide to the municipalities to treat areas of stagnant water. In the case of a positive result, OCCHD coordinates site surveys with the respective municipality. The municipalities conduct windshield surveys around the site and data is collected and graphed along with test result dates, in order to identify trends and identify effectiveness of and plan for mitigation procedures. Roles and responsibilities of each agency are outlined in the phased response plan.

-Human disease surveillance: Epidemiologists produce maps using geographic information of cases to identify clusters and possible major sources of mosquitos. This data is sent to Consumer Protection for site surveys and environmental treatments and possible habitat remediation. The Epidemiologist also gathers information on travel history and WNV exposure/risk factors of the patient. Additionally, the OCCHD epidemiologist sends out Vectorborne monitoring reports to partners providing local, state and national surveillance data. 
ISDS 2019 Conference Abstracts

\section{Results}

-Media: During the most recent seasons, OCCHD marketing and communications staff developed prevention messaging and earned more than $\$ 600,000$ in prevention messaging across the metro, to include outdoor billboard exposure, television, radio and print ads, each season. Further, OCCHD experts engage in an average of 30 media interviews each season(Television, Radio and print). Additionally, OCCD and Tyler Outdoor collaborated to present the 4D's on digital billboards across the OKC Metro area. Vital Outdoor also provides digital locations to present preventive information for 30 days, totaling $\$ 60,000$ in messaging. Finally, social media (Facebook and Twitter) outreach efforts were utilized to spread awareness to the community, including education videos reaching nearly 2,000 people.

-Vector Surveillance: Trapping sites are identified across the metro, four in Edmond and six in Oklahoma City and the mosquito season leading to 43,072 mosquitos captured and identified, of which, 33,846 were tested for WNV, a $131 \%$ increase from 2016.

-Municipality partnerships: 110 positive test pools were identified and the respective municipality is notified of the site that correlated with the positive test pool to conduct site surveys.

-Human disease surveillance: There were 6 confirmed human cases of WNV, 5 classified as Neuroinvasive and 1 classified as WNV Fever. GIS mapping is used in case investigations and habitat remediation.

\section{Conclusions}

As a single agency, this timely Vectorborne disease response would be impossible to achieve without partnerships with surrounding municipalities. Functioning as a cohesive unit, OCCHD and municipality agencies are able to set, maintain, repair and collect traps at each site, disseminate surveillance reporting information, coordinate treatment applications and investigate harborage areas. Additionally, municipalities coordinate with habitat remediation efforts. Having the resources and coordination planned for these events is critical to a timely response, especially in during an outbreak season.

OCCHD is unique in its ability to identify and sort vectors by genus and species. This capability provides detailed surveillance data and aids in preparation and planning, such as designing traps to selectively capture the most common vectors in the area. During seasons with heighted activity, the ability to have team members and partners identify geographic clusters is pivotal to timely preventive measures.

Practice based strategies to mitigate the Vectorborne disease risk to humans during an influx of increased mosquito population and positive mosquito test pools include the careful consideration of: Collaboration with outside entities in order to sustain program success; technology and GIS mapping; and strategic planning prior to the start of each season with evidence pulled form geographical analyses, vector and climate surveillance and municipality engagement.

\section{Acknowledgement}

The two municipality agencies collaborating with OCCHD are Oklahoma City Storm Water Quality (OKCSWQ) and Edmond Code Enforcement (ECE). 